\title{
Effect of the indenopyridine RTI-4587-073 (1) on feline testicle
}

\author{
F. D’Francisco ${ }^{\mathrm{a}, \mathrm{b}}$, M. López Merlo ${ }^{\mathrm{a}, \mathrm{b}}$, R. Vercellini ${ }^{\mathrm{a}, \mathrm{b}}$, P. Blanco ${ }^{\mathrm{a}, \mathrm{b}}$, C. Barbeito ${ }^{\mathrm{a}, \mathrm{b}}$, \\ C. Gobello ${ }^{\mathrm{a}, \mathrm{b}, *}$
}

${ }^{a}$ Faculty of Veterinary Sciences, National University of La Plata, Argentina

${ }^{\mathrm{b}}$ National Research Council, Argentina

\section{A R T I C L E I N F O}

\section{Keywords:}

Felid

Contraception

Non-steroidal

Testis

\begin{abstract}
A B S T R A C T
The aim of this study was to describe the seminal, histomorphological and hormonal effects of the oral indenopyridine RTI-4587-073(1) on feline testicle. Clinical side effects were also recorded. Sixty testicles of 30 adult cats that had been treated (d 0) with RTI-4587-073(l) $12.5 \mathrm{mg} / \mathrm{kg}$ PO and randomly hemiorchiectomized twice on: day $-14(n=8), 6 \mathrm{~h}(n=6), 12 \mathrm{~h}(n=8), 24 \mathrm{~h}$ $(n=6)$, day $7(n=8)$, day $14(n=6)$, day $21(n=6)$, day $35(n=6)$ or day $42(n=6)$ were studied. Before each hemiorchiectomy, fecal samples for testosterone (T) measurement were collected and the testes were grossly and ultrasound examined. This indenopyridine did not cause changes in testicular weight $(P>0.1)$, volume $(P>0.1)$, echostructure, gonadosomatic index $(P>0.1)$, fecal T concentrations $(P>0.1)$, nor clinical side effects. A severe disorganization of the cytoarchitecture of the seminiferous epithelium, sloughed cells and fluid, were observed in the $6 \mathrm{~h}$ samples up to a maximum at $24 \mathrm{~h}$. Tubular diameter $(P<0.01)$ increased twice, during the first $24 \mathrm{~h}$ and on $\mathrm{d} 35$. Germinal epithelium achieved its minimum height on $\mathrm{d} 14$ to rapidly recover thereafter. This treatment caused a significant decrease in the volume of all the seminiferous cell components, except spermatogonias. All histotological parameters normalized by the end of the study. It was concluded that RTI-4587-073(l) severely disrupted spermatogenesis during the first $24 \mathrm{~h}$ after treatment returning to normality in approximately one spermatic cycle without clinical side effects.
\end{abstract}

\section{Introduction}

Domestic felids (Felis catus) are extremely prolific breeders, they mate from early puberty up to death and, if kept indoors or at latitudes higher than $35^{\circ}$, throughout the year (Faya et al., 2011). Thus, contraception - reversible reproduction control - is often required for genetically valuable domestic or wild cats. As in most other mammalian species, feline contraception is mainly targeted towards females and progestins are commonly used provoking, sometimes, severe reproductive and general health side effects (Munson, 2006). Although both steroidal and non-steroidal approaches have been assessed in males, controlling spermatogenesis probed to be a more complex objective than preventing ovulation in females. Furthermore, most male contraceptive protocols have shown a variable lag time to achieve azoospermia as well as an unpredictable return to fertility (Bowen, 2008). Safe, rapid and efficient pharmaceutical compounds are still needed to temporally control the undesired reproduction in male felids. If tom cats were rendered temporarily sterile, female reproductive health could also be preserved.

The group of indenopyridine derivatives, which were originally developed as antihistamine drugs, has shown antispermatogenic

\footnotetext{
* Corresponding author at: Laboratory of Reproductive Physiology, Faculty of Veterinary Sciences, National University of La Plata, 60 y 118 . La Plata CC 296 (B 1900 AVW), Argentina.

E-mail address: cgobello@fcv.unlp.edu.ar (C. Gobello).
} 
effects in rats (Hodel and Suter, 1978), stallions (Pozor et al., 2013) and dogs (Chang et al., 2002) without overt side effects, toxicity nor mutagenic potential (Fail et al., 2000; Mruk, 2008). Studies in laboratory animals showed that the primary target of indenopyridines is the Sertoli cell, resulting in disruption of Sertoli - germ cell adhesion (Hild et al., 2001, 2007b; Koduri et al., 2008).

While in monkeys indenopyridines induced reversible severe oligoasthenozoospermia (Hild et al., 2007a), irreversible infertility was described in rats (Hild et al., 2004). In horses and dogs indenopyridines induced a rapid (few days) and transient inhibition of spermatogenesis increasing the number of immature germ cells in ejaculates (Chang et al., 2002; Hodel and Suter, 1978; Pozor et al., 2013, 2014). Histologically the seminiferous tubuli appeared emptied of spermatides and spermatocytes (Pozor et al., 2013). Although the testicular effects described in these four species make indenopyridines a promising candidate for non-steroidal, oral male contraception in felids, nothing is known about their effect, reversibility and tolerability in this species (Munson, 2006).

The compound RTI-4587-073, formerly called CDB-4022, is a new indenopyridine derivative, which is a mixture of L and Disomers. The L-isomer exhibits a particularly strong anti- spermatogenic activity (Pozor et al., 2013) and, therefore, was chosen for this feline trial. Thus, the aim of this study was to describe the ultrasonographic, histomorphological and hormonal effects of oral indenopyridine RTI-4587-073(1) on feline testicle. Secondly, some epididymal semen characteristics and clinical side effects of the drug were also recorded in the treated cats.

\section{Material and methods}

\subsection{Experimental protocol}

Sixty testicles of 30, 1.5-5 years old, domestic short- hair, fertile cats were included in this study. The males were born in our Institutional Colony, exposed to $14 \mathrm{~h}$ of light per day in three $4 \times 4 \mathrm{~m}$ enriched rooms, fed commercial food and water ad libitum. The animals were treated (day 0) with RTI-4587-073(l) $12.5 \mathrm{mg} / \mathrm{kg}$ PO and randomly hemiorchiectomized twice on: day -14 ( $n=8$ ), $6 \mathrm{~h}$ $(n=6), 12 \mathrm{~h}(n=8)$, day $1(n=6)$, day $7(n=8)$, day $14(n=6)$, day $21(n=6)$, day $35(n=6)$ or day $42(n=6)$. The calculated dose was dissolved in $2 \mathrm{cc}$ of saline and administered by a naso-esophageal tube after mild sedation. The dose and dosing scheme were selected according to studies in other mammals (Hild et al., 2001; Pozor et al., 2013) and to our own pilot trials in cats.

Before each hemiorchiectomy, the felids were weighed, fecal samples were collected and the testes were grossly and ultrasound examined in a way that each animal had 2 random evaluation time points on the same days of surgeries. The appearance of clinical side effects, such as lack or diminished appetite, vomiting or other digestive signs, changes in body weight or sexual or general behavior, was also recorded in all the animals. This study was reviewed and approved by the Animal Care and Use Committee of the Veterinary School of the National University of La Plata and all experiments were conducted under the guidelines established in The Guide for The Care and Use of Laboratory Animals, USA.

\subsection{Ultrasound evaluations}

Ultrasound examinations of the testes were undertaken by a single experienced evaluator using a real time B-mode ultrasound machine (Toshiba Nemio XG, Japón) with a $14 \mathrm{MHz}$ lineal transducer. All machine settings were established at the first examination according to best image quality and remained unaltered for all remaining examinations. Acoustic gel was applied to the transducer and coupled directly to the clipped scrotum with minimum pressure to obtain the images. The testes were imaged in the sagittal and transverse planes. Testicular dimensions were obtained from frozen images, using the ultrasound calipers and the total testicular volume was calculated as described by Lin et al. (2009). Ecogenicity and homogeneity of the parenchyma were also assessed at each time point.

\subsection{Fecal collection, extraction and hormone determinations}

Fecal samples were collected at the time points of hemiorchiectomies (see below) and frozen for testosterone (T) determination. Fecal steroids were extracted using the methods described by Faya et al. (2013) and T (ng/ml) was determined using electrochemiluminescence immunoassay (Elecsys Testo II, Roche Diagnostics, Mannheim, Germany). Inter- and intra-assay coefficients of variation of the assays were $<10 \%$ and sensitivity was $0.025 \mathrm{ng} / \mathrm{mL}$. All fecal data were expressed on a wet-weight basis

\subsection{Hemiorchiectomies}

All the cats were randomly hemiorchiectomized twice according to García Romero et al. (2012) in a way that a total of 60 testes were obtained. Briefly, the animals were premedicated with atropine sulfate, (Atropine Sulfate, John Martin; 0.04 mg/kg SC), acepromazine maleate (Acedan, Holliday; $0.03 \mathrm{mg} / \mathrm{kg} \mathrm{SC}$ ), and butorphanol (Torbutol Plus, Fort Dodge; $0.2 \mathrm{mg} / \mathrm{kg} \mathrm{IM}$ ), anesthesia was induced with sodium thiopental (Pentovet TM, Richmond; $8 \mathrm{mg} / \mathrm{kg}$ IV) in all of the animals. Once the cats were endotracheally intubated, anesthesia was maintained with isoflurane and oxygen in a closed system. Each testis was removed through a longitudinal midscrotal incision using sterile surgical procedures and a closed tunic technique, finally the spermatic cord was ligated with 3.0 vicryl before transection. After surgery ketoprofen (Ketofen ${ }^{\circledast}$, Fort Dodge; $1 \mathrm{mg} / \mathrm{kg}$ ) were injected SC (once) and then orally every $24 \mathrm{~h}$ during 4 additional days. Then, all the cats were placed for adoption. 


\subsection{Gross and histological examination of the testes}

Immediately after surgical removal, the testes were measured (length, width and depth; $\mathrm{cm}$ ) and weighed $(\mathrm{g})$ and gonadosomatic index (\%; Franca and Godinho, 2003) was also calculated.

Sperm was recovered by the epididymal tail sectioning using a scalpel blade. Spermatozoa morphology was evaluated by Giemsa stain, under 1000X magnification using bright field microscopy (Valiente et al., 2014).

The testes, were sectioned longitudinally, placed in Bouin's fixative for $12 \mathrm{~h}$ and then changed to alcohol 70 and processed routinely with paraffin embedding. After processing, $5 \mu \mathrm{m}$ serial sections were cut, mounted on slides, dried, deparaffinized in xylene, rehydrated in graded ethanol solutions and stained with hematoxylin and eosin. Histological images were obtained from a microscope (Olympus BX50; 10X and 40X) through an attached video camera (Sony DXC 151) and digitalized in a 24 bit true color TIFF format. Twenty tubular profiles, which were round or nearly round, were chosen randomly and measured for each animal. The tubular diameter of seminiferous tubules $(\mu \mathrm{m})$ and the germinal epithelium height $(\mu \mathrm{m})$ were measured by planimetry (Image Pro Plus, Media Cybernetics, Silver Spring, MA, USA). The volume $(\mathrm{cm} 3)$ of the different testicular tissue components was determined by stereology using a light microscopy and a 441-intersection grid placed on 40X tissue pictures. For this, 15 fields were chosen randomly (6615 points) for each testis. Points were classified as one of the following: spermatogonia, primary and secondary spermatocytes, round spermatids, elongated spermatids, spermatozoa, Sertoli and Leydig cells, intertubular compartment, and cellular debris. Finally, the proportion of each element was calculated using the whole testicular volume as previously described by Carranza et al. (2015).

\subsection{Statistical analysis}

Normality of distribution of results was tested using a Shapiro - Wilk normality test. All hormonal, gross and microscopical variables were compared among time points by Kruskall-Wallis test followed by a Dunn's multiple comparisons test. In all the cases descriptive data were expressed as mean \pm SEM and $P$ values $>0.05$ were considered significant.

\section{Results}

Neither gross testicular parameters including weight $(P>0.1)$, volume $(P>0.1)$ and gonadosomatic index $(P>0.1)$ nor body weight $(P>0.1$ ) were affected by RTI-4587-073 (1) treatment at the different evaluation time points (Table 1). Although epididymal spermatozoa morphology was normal in all the cases, giant multinucleated cells appeared in the semen samples during the first $24 \mathrm{~h}$ after treatment (Fig. 1).

Histological testicular structure was normal on day -14 (Fig. 2; A) and a severe disorganization of the cytoarchitecture of the seminiferous epithelium was observed as early as in the $6 \mathrm{~h}$ samples reaching a maximum impairment at $24 \mathrm{~h}$, with the presence of vacuolization, immature germ and multinucleated cells as well as intratubular fluid (Fig. 2; C). During the first $24 \mathrm{~h}$ several seminiferous tubules were in the process of sloughing or had already sloughed material within the lumen occluding the tubules along with the fluid and cellular detritus $(P<0.01$; Fig. 2, C and D; Table 2). These abnormalities gradually disappeared up to the end of the study (Fig. 2, F, H, I) and could not be evidenced through their echostructure which was normal at all time points (Mattoon and Nyland, 1995).

Seminiferous tubular diameter $(P<0.01$; Fig. 3, Inset) increased in detriment to the interstitium $(P<0.01$; Table 2) during the first $24 \mathrm{~h}$ and, then, reached its peak value on $\mathrm{d} 35$. Finally this diameter showed a tendency to return to initial values up to the end of the study.

Germinal epithelium height could not be measured during the first $24 \mathrm{~h}$ after treatment due to the major disorganization of its cytoarchitecture (Fig. 3). This epithelium presented its minimum height on day 14 to rapidly recover to reach peak values on day 21 and, thereafter, it maintained that height (Fig. 3). Although the volume occupied by spermatogonia did not varied throughout the study $(P>0.1)$ the volume of the primary spermatocytes decreased during the same period $(P<0.01$; Table 2$)$. This diminution was even more marked in the case of secondary spermatocytes $(P<0.01)$, round $(P<0.01)$ and elongated $(P<0.01)$ spermatids as well as luminal spermatozoa $(P<0.01)$ which reappeared just before the end of the study on day 42 . Conversely, the volume

\section{Table 1}

Morphometric data of the 30 male cats which had been administered (d 0) a single PO dose of $12.5 \mathrm{mg} / \mathrm{kg}$ RTI-4587-073(l) and randomly hemiorchiectomized twice on: day $-14(n=8), 6 \mathrm{~h}(n=6), 12 \mathrm{~h}(n=8)$, day $1(n=6)$, day $7(n=8)$, day $14(n=6)$, day $21(n=6)$, day $35(n=6)$ or day $42(n=6)$.

\begin{tabular}{|c|c|c|c|c|c|c|c|c|c|}
\hline & $\mathrm{d}-14$ & h 6 & h 12 & h 24 & d 7 & d 14 & d 21 & d 35 & d 42 \\
\hline Body weight (kg) & $4.83 \pm 0.28$ & $5.35 \pm 0.35$ & $4.90 \pm 0.49$ & $4.40 \pm 0.20$ & $4.74 \pm 0.28$ & $4.48 \pm 0.16$ & $4.65 \pm 0.35$ & $4.93 \pm 0.19$ & $4.47 \pm 0.32$ \\
\hline Testis weight (g) & $1.95 \pm 0.12$ & $1.76 \pm 0.26$ & $1.85 \pm 0.24$ & $1.93 \pm 0.04$ & $1.87 \pm 0.35$ & $1.87 \pm 0.34$ & $1.77 \pm 0.21$ & $1.89 \pm 0.47$ & $1.92 \pm 0.55$ \\
\hline $\begin{array}{l}\text { Testicular volumen } \\
\quad\left(\mathrm{cm}^{3}\right)\end{array}$ & $2.69 \pm 1.43$ & $2.54 \pm 0.40$ & $2.10 \pm 0.29$ & $2.76 \pm 0.29$ & $2.67 \pm 0.97$ & $1.85 \pm 0.92$ & $2.28 \pm 0.62$ & $2.43 \pm 0.66$ & $2.54 \pm 0.68$ \\
\hline $\begin{array}{l}\text { Gonadosomatic } \\
\text { index (\%) }\end{array}$ & $0.08 \pm 0.01$ & $0.08 \pm 0.08$ & $0.08 \pm 0.01$ & $0.09 \pm 0.00$ & $0.08 \pm 0.01$ & $0.08 \pm 0.01$ & $0.08 \pm 0.01$ & $0.08 \pm 0.01$ & $0.08 \pm 0.01$ \\
\hline
\end{tabular}




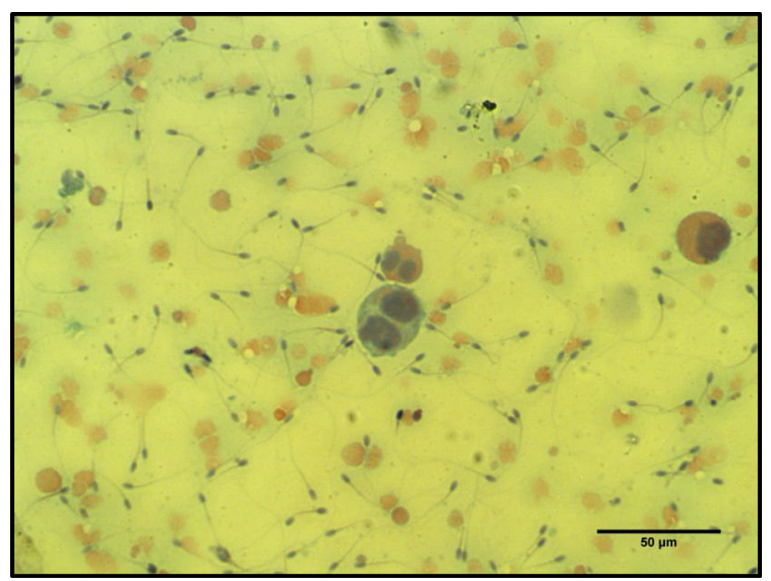

Fig. 1. Giant multinucleated cell (Giemsa stain, 1000X) found in epididymal sperm during the first $24 \mathrm{~h}$ after RTI-4587-073(l) administration in most of the male cats of Table 1 .

occupied by Sertoli cells increased $24 \mathrm{~h}$ after treatment to significantly decrease up to day 14 when it began to return to pretreatment volume ( $P<0.01$; Table 2 ).

Although, the volume occupied by Leydig cells decreased in the first post treatment observations $(P<0.01$; Table 2), fecal T concentrations did not vary during the same period (data not shown; $P>0.1$ ). Finally, this indenopyridine did not cause general, digestive or behavioral side effects in any animal during the study period.

\section{Discussion}

Widespread use of contraceptives has been limited in male felids by safety concerns and the lack of effective choices. In spite that indenopyridines have shown antispermatogenic effects in several mammals, there are species differences which justify such study in the feline species. Up to the authors' knowledge, this is the first report that describes their effects in cats.

In line with most articles in rats (Hild et al., 2001; Koduri et al., 2008) and monkeys (Hild et al., 2007a) but unlike to those in horses (Pozor et al., 2014), T did not seem to be affected by RTI-4587-073 (1) treatment in these felids. While suppression of T and, therefore, libido would have been desirable in domestic cats, $\mathrm{T}$ dependent changes in behavior and phenotype are definitively a disadvantage for wild felids as they may alter their group hierarchy as well as their secondary sexual characteristics. Conversely, the volume occupied by Leydig cells was decreased in the post treatment observations. In this respect, it should be borne in mind that in this trial, fecal determinations could have masked transient serum T variations that could have been evidenced in blood samples. In line with a horse report using the same pharmacological protocol (Pozor et al., 2014), total testicular volume did not change throughout the days of this feline trial.

The rapidity of histological effects was one of the most interesting findings of the present antispermatogenic protocol. Similar rapid effects of treatment with RTI-4587-073(1) on testicular tissue were previously described in monkeys, horses, dogs and rats (Chang et al., 2002; Hild et al., 2001, 2007a; Pozor et al., 2014).

Particularly, the seminiferous epithelium vacuolization and sloughing into the tubular lumen causing severe tubular disorganization is a common histopathological observation associated with Sertoli cell injury after exposure to testicular toxicants (Johnson, 2015; Vidal and Whitney, 2014). The multinucleated giant cells found both in the epithelium and semen are thought to arise from fusion of degenerate spermatids with abnormal intercellular bridges (Vidal and Whitney, 2014). This kind of cells was also previously observed in rats, horses and dogs treated with bisdiamines (Chang et al., 2002; Hild et al., 2001; Pozor et al., 2013).

Specifically, indenopyridines alter Sertoli cells - germ cell adherents' junction proteins leading to a massive germ cell loss (Koduri et al., 2008). As Sertoli cell junctions are an essential component of the blood-testis barrier required for normal spermatogenesis they have been considered a target for male contraceptive development (Lee et al., 2009). In this study, the injury in Sertoli cells could, not only, explain the early increase in their own volume but also the augmentation of the whole tubular diameter associated with fluid production on day 1 (Vidal and Whitney, 2014).

As expected, in the presence of these severe histological findings almost all cellular components of the germinal epithelium were significantly affected by the indenopyridine treatment. Importantly, the major shortage of germinal cells occurred at the level of spermatocytes and spermatids evidencing a severe loss of the outer layers.

Before the treatment on day -14 all histomorphometric parameters were in line with those previously described for the species (Franca and Godinho, 2003; Siemieniuch and Wocławek-Potocka, 2007). The early increase in the seminiferous tubule diameter found in this study has also been associated with the tubular fluid production after Sertoli cells toxicant administration (Vidal and Whitney, 2014). The late augmentation of this diameter on day 35 could be explained as a rebound recovery of spermatogenesis at that time point (Meisami et al., 1994).

The whole height of the germinal epithelium rapidly decreased to two layers of cells on day 14 to recover to values even higher 

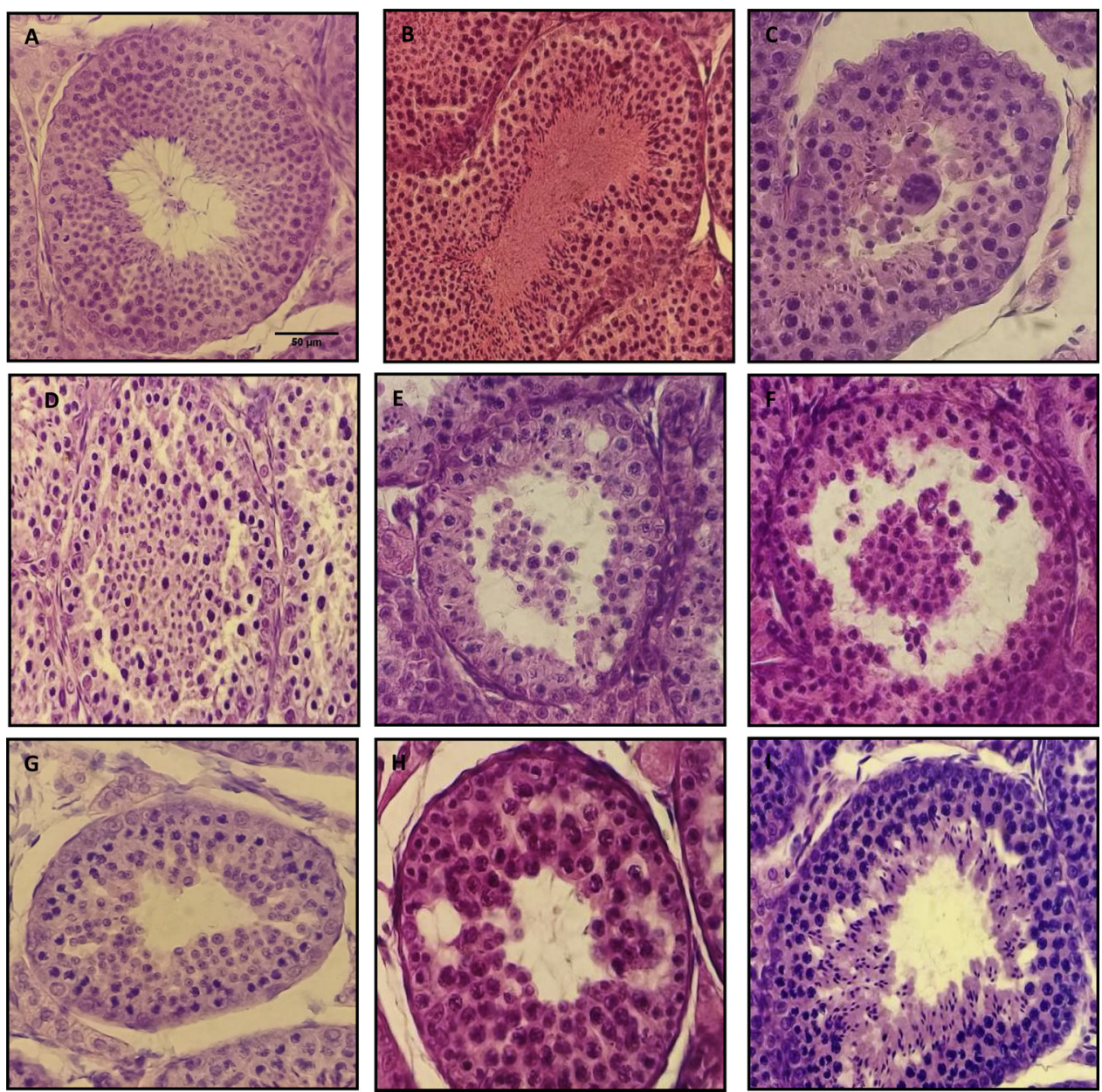

Fig. 2. Seminiferous tubules of the cats and experiment of Table 1 at different time points: -14 (A), $6 \mathrm{~h}$ (B), $12 \mathrm{~h}$ (C), day 1 (D), day 7 (E), day 14 (F), day $21(\mathrm{G})$, day $35(\mathrm{H})$ and day 42 (I) All sections were stained with hematoxylin and eosin (40X).

Notice normal germinal epithelium and spermatozoa at the luminal border (A), fluid in the lumen of a seminiferous tubule (B), seminiferous epithelium sloughing into the lumen with a giant multinucleated cell in the center (C), severe disorganization of the seminiferous epithelium with occluding detritus filling the lumen (D), epithelium sloughing is finishing and some vacuoles are appearing, luminal detritus is diminishing (E), thin germinal epithelium with frayed luminal border and some detritus in the lumen (F), quite recovered germinal epithelium with still some vacuoles and a clean lumen (G), recovered germinal epithelium height with some vacuoles $(\mathrm{H})$, fully recovered germinal epithelium with abundant elongated spermatids at the luminal border (I).

than the pretreatment ones a week later. The tubular presence of unaffected spermatogonias throughout the study period guaranteed the recovery of spermatogenesis and, therefore, the reversibility of this feline pharmacological protocol. Indenopyridines have variably produced either a reversible (in dogs, monkeys and horses; Chang et al., 2002; Hild et al., 2007a; Pozor et al., 2013) or irreversible (in rats; Hild et al., 2004) effect on fertility. Surprisingly, the lowest dose $-2.5 \mathrm{mg} / \mathrm{kg}$ - among these trials, was the one which caused irreversible damage in laboratory rodents (Hild et al., 2004) which suggests a species - specific effect. In these domestic cats, reversibility of the germinal epithelium occurred at approximately one feline spermatic cycle period (Franca and Godinho, 2003). Similarly, in indenopyridine treated horses and dogs, reversible spermatogenesis was noted 6 and 7 weeks after its administration, respectively (Chang et al., 2002; Pozor et al., 2014).

Considering the rapid and severe histological changes as well as their reversibility it would be of interest in the future to in vivo test fertility during the peak indenopyridine effect and also after spermatogenic recovery so that rational, single or serial - doses protocols could be proposed for felids.

Finally and in keeping with publications in rodents (Fail et al., 2000) no observable adverse effects such as body weight loss or digestive problems were found in the treated animals, suggesting that this compound might not have general toxicity in felids.

It was concluded that RTI-4587-073(1) represents a rapid, effective, reversible and safe non-steroid antispermatogenic oral protocol for male cat contraception as it severely disrupted spermatogenesis during the first $24 \mathrm{~h}$ after treatment returning to normality in approximately one spermatic cycle. Further refinement, including repeated doses and surveillance for toxicological and long-term adverse effects, of these indenopyridine derivatives should be carried out before their field implementation could be widely 


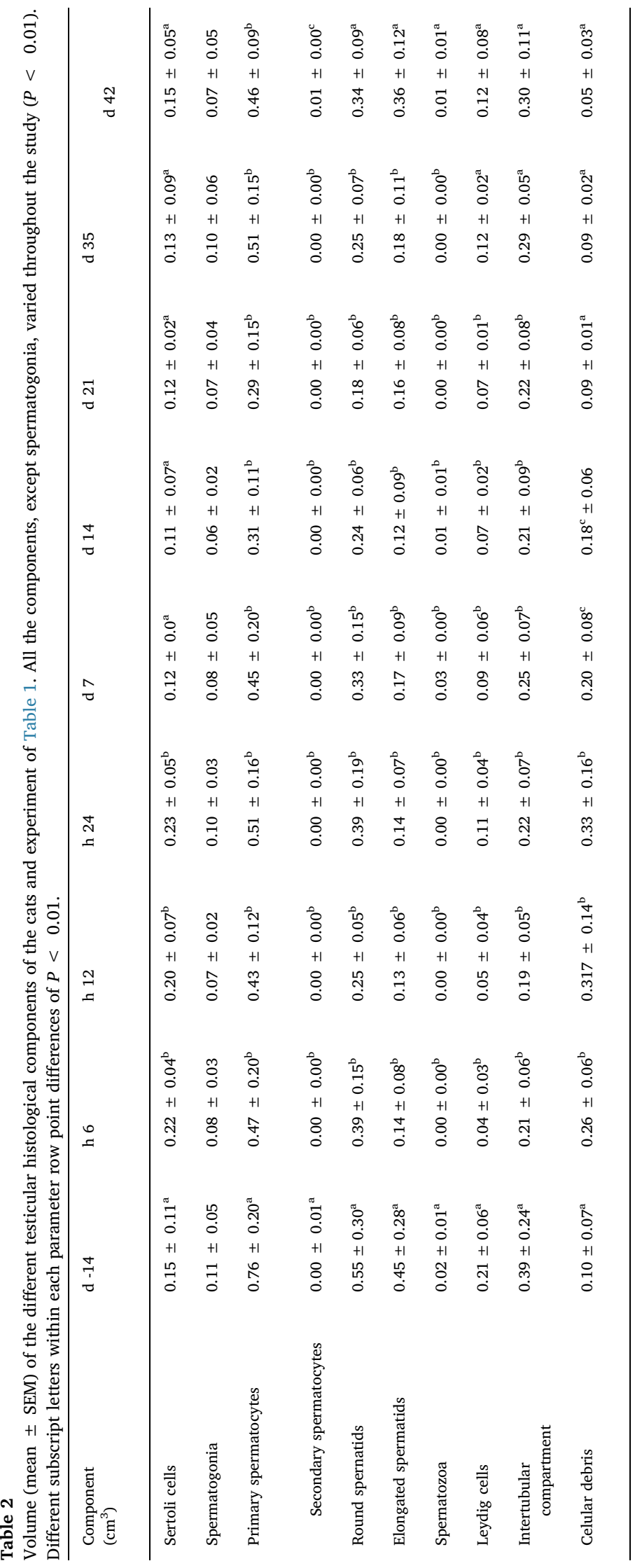




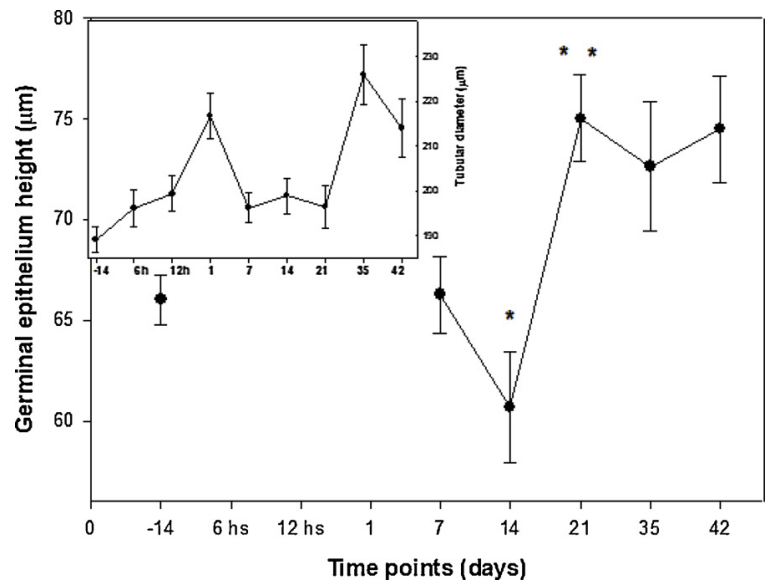

Fig. 3. Testicular germinal epithelium height and tubular diameter (mean \pm SEM; Inset) of the cats and experiment of Table 1. Germinal epithelium height could not be measured during the first $24 \mathrm{~h}$ after treatment. Asterisks point differences of $P<0.05$.

recommended.

\section{Conflict of interest}

The authors do not have any financial nor personal relationships with other people or organizations that could inappropriately influence the study.

\section{Acknowledgments}

This work was partially supported by the Teaching Incentive Program of NULP (V233). RTI-4587-073(1) was kindly provided by the Research Triangle Institute, Research Triangle Park, NC, USA. FDF, MLM, RV are a Research Fellows, and PB, CB and CG are Career Scientists of CONICET, Argentina.

\section{References}

Bowen, R.A., 2008. Male contraceptive technology for nonhuman male mammals. Anim. Reprod. Sci. 105, 139-143.

Carranza, A., Faya, M., Fernandez, P., Barbeito, C., Gobello, C., 2015. Histologic effect of a postnatal slow-release GnRH agonist on feline gonads. Theriogenology 25, 1097-1100.

Chang, C.L., Fung, H.P., Lin, Y.F., Kuo, C.Y., Chien, C.W., 2002. Indenopyridine hydrochloride induced testicular spermatogenesis failure with high seminal alkaline phosphatase levels in male dog. Biol. Pharm. Bull. 25, 1097-1100.

Fail, P.A., Anderson, S.A., Cook, C.E., 2000. 28-day toxicology test: indenopyridine RTI 4587-056 in male Sprague-Dawley rats. Reprod. Toxicol. 14, 265-274.

Faya, M., Carranza, A., Priotto, M., Abeya, M., Diaz, J., Gobello, C., 2011. Domestic queens under natural temperate photoperiod do not manifest seasonal anestrus. Anim. Reprod. Sci. 129, 78-81.

Faya, M., Carranza, A., Miotti, R., Ponchón, T., Furlan, P., Gobello, C., 2013. Fecal estradiol-17 beta and testosterone in prepubertal domestic cats. Theriogenology 80, 584-586.

Franca, L.R., Godinho, C., 2003. Morphometry, seminiferous epithelium cycle length, and daily sperm production in domestic cats (Felis catus). Biol. Reprod. 68, $1554-1561$.

Hild, S.A., Reel, J.R., Larner, J.M., Blye, R.P., 2001. Disruption of spermatogenesis and Sertoli cell structure and function by the indenopyridine CDB-4022 in rats. Biol. Reprod. 65, 1771-1779.

Hild, S.A., Attardi, B.J., Reel, J.R., 2004. The ability of a gonadotropin-releasing hormone antagonist, acyline, to prevent irreversible infertility induced by the indenopyridine, CDB-4022, in adult male rats: the role of testosterone. Biol. Reprod. 71, 348-358.

Hild, S.A., Marshall, G.R., Attardi, B.J., Hess, R.A., Schlatt, S., Simorangkir, D.R., et al., 2007a. Development of 1-CDB-4022 as a non steroidal male oral contraceptive: induction and recovery from severe oligospermia in the adult male cynomolgus monkey (Macaca fascicularis). Endocrinology 148 , $1784-1796$.

Hild, S.A., Reel, J.R., Dykstra, M.J., Mann, P.C., Marshall, G.R., 2007b. Acute adverse effects of the indenopyridine CDB-4022 on the ultrastructure of Sertoli cells, spermatocytes, and spermatids in rat testes: comparison to the known Sertoli cell toxicant Di-n-pentylphthalate (DPP). J. Androl. 28 , 621-629.

Hodel, C., Suter, K., 1978. Reversible inhibition of spermatogenesis with an indenopyridine (20-438). Arch. Toxicol. (Suppl. 1), 323-326.

Johnson, K.J., 2015. Testicular histopathology associated with disruption of the Sertoli cell cytoskeleton. Spermatogenesis 19 , e979106.

Koduri, S., Hild, S.A., Pessaint, L., Reel, J.R., Attardi, B.J., 2008. Mechanism of action of 1-CDB-4022, a potential nonhormonal male contraceptive, in the seminiferous epithelium of the rat testis. Endocrinology 149, 1850-1860.

Lee, N.P., Wong, E.W., Mruk, D.D., Cheng, C.Y., 2009. Testicular cell junction: a novel target for male contraception. Curr. Med. Chem. 16 , $906-915$.

Lin, C.C., Huang, W.J., Chen, K.K., 2009. Measurement of testicular volume in smaller testes: how accurate is conventional orchidometer? Androl. 30 (685-), 689.

Mattoon, J., Nyland, T., 1995. Ultrasonography of the genital system. In: Nyland, T., Mattoon, J. (Eds.), Veterinary Diagnostic Ultrasound. Saunders, W.B., Philadelphia, pp. 141-164.

Meisami, E., Najafi, A., Timiras, P.S., 1994. Enhancement of seminiferous tubular growth and spermatogenesis in testes of rats recovering from early hypothyroidism: a quantitative study. Cell Tissue Res. 275, 503-511.

Mruk, D.D., 2008. New perspectives in non-hormonal male contraception. Trends Endocrinol. Metab. 19, 57-64.

Munson, L., 2006. Contraception in felids. Theriogenology 1, 126-134.

Pozor, M.A., Macpherson, M.L., Mc Donnell, S.M., Nollin, M., Roser, J.F., Love, C., et al., 2013. Indenopyride derivative RTI-4587-073(1): a candidate for male contraception in stallions. Theriogenology 80, 1006-1016. 
Pozor, M., Zambrano, G., Roser, J., Hess, R., Runyon, S., Runcan, E., et al., 2014. Acute and chronic effects of a contraceptive compound RTI-4587-073(1) on testicular histology and endocrine function in miniature horse stallions. Reprod. Domest. Anim. 49, 392-402.

Romero, G.G., Barbeito, C., Fernandez, P., Gimeno, E., Gobello, C., 2012. Unilateral orchidectomy in mature cats is not followed by compensatory hypertrophy. Reprod. Domest. Anim. 6 (Suppl. 47), 226-228.

Siemieniuch, M.J., Wocławek-Potocka, I., 2007. Morphological features of the seminiferous epithelium in cat (Felis catus, L. 1758) testes. J. Reprod. Dev. 53, $1125-1130$.

Valiente, C., Arauz, S., de la sota, P., Gobello, C., 2014. Ejaculation training, seminal alkaline phosphatase and preservation through cooling in a milk-based extender in domestic cats. J. Feline Med. Surg. 16, 312-316.

Vidal, J.D., Whitney, K.M., 2014. Morphologic manifestations of testicular and epididymal toxicity. Spermatogenesis 3, e979099. 